\title{
Milliliter per Kilogram per Minute
}

National Cancer Institute

\section{Source}

National Cancer Institute. Milliliter per Kilogram per Minute. NCI Thesaurus. Code C73760.

A dose calculation unit expressed in milliliter(s) per kilogram per period of time equal to sixty seconds. 